\title{
A Wave Splitter with Simple Structure Based on Biaxial Anisotropic Medium
}

\author{
Chushun Zhang, Hui Huang, Luchen Huang, Yaru Dong, and Xiaoyu Liu
}

School of Electrical Engineering, Beijing Jiaotong University, Beijing 100044, China

Correspondence should be addressed to Hui Huang; hhuang@bjtu.edu.cn

Received 12 March 2017; Revised 20 April 2017; Accepted 27 April 2017; Published 23 May 2017

Academic Editor: Giuseppe Castaldi

Copyright (c) 2017 Chushun Zhang et al. This is an open access article distributed under the Creative Commons Attribution License, which permits unrestricted use, distribution, and reproduction in any medium, provided the original work is properly cited.

\begin{abstract}
A TE/TM wave splitter based on biaxial anisotropic slab is designed. According to the total reflection and total transmission of TE/TM waves incident from isotropic medium into biaxial anisotropic medium, we propose a wave splitter with simple structure and little limitation on both constitutive parameters and incident angle. All the results are verified by simulation.
\end{abstract}

\section{Introduction}

The studies of metamaterial have been the research focus in the field of electromagnetic and materials since Veselago proposed left-handed medium in 1968 [1] and Smith et al. made the first metamaterial sample which has negative index with permittivity and permeability in 2001 [2]. It is well known that the propagations and characteristics of metamaterial are significantly different from those of isotropic materials [3-15]. Metamaterial has many unusual properties and may lead to important applications, such as absorber [16], wave splitter [17], and subwavelength rectangular cavity [18].

Theoretically, metamaterial can be considered biaxial anisotropic medium; that is, its permittivity and permeability are both diagonal tensors:

$$
\begin{aligned}
& \overline{\bar{\varepsilon}}=\left[\begin{array}{ccc}
\varepsilon_{x} & 0 & 0 \\
0 & \varepsilon_{y} & 0 \\
0 & 0 & \varepsilon_{z}
\end{array}\right], \\
& \overline{\bar{\mu}}=\left[\begin{array}{ccc}
\mu_{x} & 0 & 0 \\
0 & \mu_{y} & 0 \\
0 & 0 & \mu_{z}
\end{array}\right] .
\end{aligned}
$$

Generally, $\varepsilon_{x} \neq \varepsilon_{y} \neq \varepsilon_{z}$ and $\mu_{x} \neq \mu_{y} \neq \mu_{z}$. The studies of biaxial anisotropic medium have received much interest and attention. Reference [19] has shown that anomalous negative refraction can occur at an interface associated with a metamaterial, which does not necessarily require that all tensor elements of $\overline{\bar{\varepsilon}}$ and $\overline{\bar{\mu}}$ have negative values. The propagation of plane-wave in biaxial anisotropic medium with singlesheeted hyperboloid dispersion relation is investigated in [20]. Characteristics of surface wave in biaxial anisotropic left-handed materials are discussed in [21]. Negative refraction and cross polarization effects of biaxial anisotropic medium are summarized in [22]. The double-reflection and light interference pattern in a biaxial anisotropic thin film is numerically investigated in [23]. Under such circumstances, this paper focuses on the application of biaxial anisotropic medium.

Because of different dispersion relations of TE waves and TM waves in biaxial anisotropic medium, they propagate in different directions and phase velocities. Thus, the propagation of electromagnetic waves can be controlled by designing constitutive parameters. In this paper, we present the theoretical analysis of a wave splitter based on biaxial anisotropic medium.

Nowadays, available technology can separate electromagnetic waves by their different power, mode, or frequency according to different engineering applications [24-28]. The wave splitter based on biaxial anisotropic medium we design in this paper can separate electromagnetic waves by polarization. More specifically, this slab with specific constitutive parameters can make TE waves totally reflected and TM waves totally transmitted, while, with the other specific 
TABLE 1: Conditions of total reflection of TE waves.

\begin{tabular}{ll}
\hline The range of constitutive parameters & The condition of total reflection \\
\hline & $\begin{array}{l}\text { (a) If } \varepsilon_{z} \mu_{x}>\varepsilon_{0} \mu_{0} \text {, there is no total reflection at the } \\
\text { boundary } \\
\text { (b) If } \varepsilon_{z} \mu_{x}<\varepsilon_{0} \mu_{0} \text {, total reflection occurs when incident } \\
\text { angle } \theta_{i}>\text { critical angle } \theta_{c} \text {, where } \\
\theta_{c}=\arcsin \sqrt{\varepsilon_{z} \mu_{x} / \varepsilon_{0} \mu_{0}}\end{array}$ \\
\begin{tabular}{ll} 
(A) $\mu_{x} \mu_{y}>0 \cap \varepsilon_{z} \mu_{y}>0$ & Total reflection occurs for all TE waves \\
\hline (B) $\mu_{x} \mu_{y}>0 \cap \varepsilon_{z} \mu_{y}<0$ & (a) If $\varepsilon_{z} \mu_{x}>\varepsilon_{0} \mu_{0}$, total reflection occurs for all TE \\
& waves no matter what incident angle $\theta_{i}$ is \\
(C) $\mu_{x} \mu_{y}<0 \cap \varepsilon_{z} \mu_{y}<0$ & (b) If $\varepsilon_{z} \mu_{x}<\varepsilon_{0} \mu_{0}$, total reflection occurs when incident \\
& angle $\theta_{i}<$ critical angle $\theta_{c}$
\end{tabular} \\
\hline
\end{tabular}

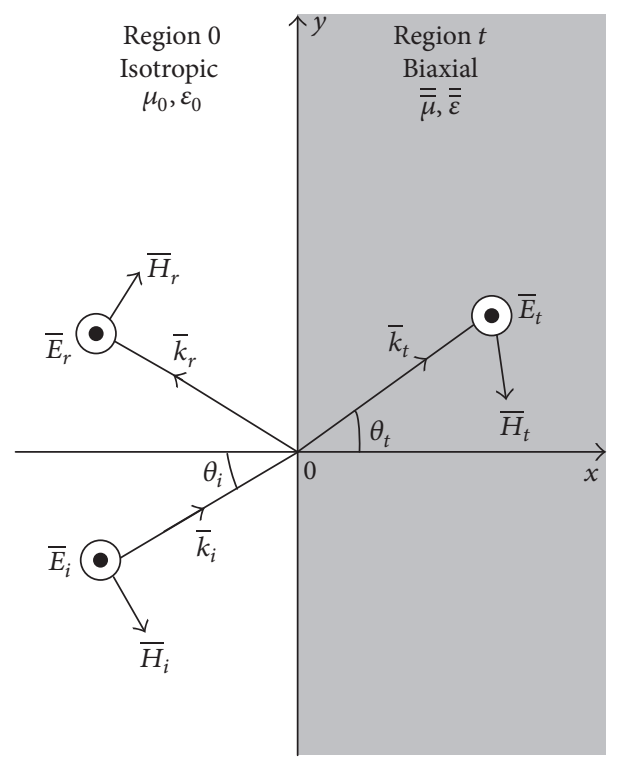

FIGURE 1: Reflection and transmission of a TE wave at an interface between a semi-infinite isotropic medium (region 0 ) and a biaxial anisotropic medium (region $t$ ).

constitutive parameters, we can get TM waves totally reflected and TE waves totally transmitted. Compared with traditional wave splitters, such a wave splitter with simple structure has little limitation on both constitutive parameters and incident angle. Meanwhile, it can be designed with any chirality of biaxial anisotropic medium and any width. This provides a theoretical basis to the engineering practice.

The paper is organized as follows: in Section 2, we present a brief review on the total reflection and total transmission in biaxial anisotropic medium. In Section 3, we discuss two ways to separate TE waves and TM waves. Finally in Section 4, we summarize the advantages of this TE/TM wave splitter.

\section{Theoretical Analysis}

2.1. Total Reflection of TE Waves. Considering the configuration in Figure 1, a TE wave is incident from a semi-infinite isotropic medium into a biaxial anisotropic medium at an oblique angle $\theta_{i}$. Region 0 is the isotropic medium, with permittivity $\varepsilon_{0}$ and permeability $\mu_{0}$. Region $t$ is the biaxial anisotropic medium, with permittivity $\overline{\bar{\varepsilon}}$ and permeability $\overline{\bar{\mu}}$ which are shown in (1) and (2), respectively.

For Figure 1, with wave vectors $\bar{k}_{i}^{\mathrm{TE}}=\hat{x} k_{0 x}^{\mathrm{TE}}+\hat{y} k_{y}^{\mathrm{TE}}$ (incident) and $\bar{k}_{r}^{\mathrm{TE}}=-\widehat{x} k_{0 x}^{\mathrm{TE}}+\widehat{y} k_{y}^{\mathrm{TE}}$ (reflection) in the isotropic medium and $\bar{k}_{t}^{\mathrm{TE}}=\hat{x} k_{t x}^{\mathrm{TE}}+\widehat{y} k_{t}^{\mathrm{TE}}$ (transmission) in the biaxial anisotropic medium, the dispersion relations can be expressed as

$$
\begin{aligned}
& \left(k_{0 x}^{\mathrm{TE}}\right)^{2}+\left(k_{y}^{\mathrm{TE}}\right)^{2}=\omega^{2} \varepsilon_{0} \mu_{0}, \\
& \frac{\left(k_{t x}^{\mathrm{TE}}\right)^{2}}{\mu_{y}}+\frac{\left(k_{y}^{\mathrm{TE}}\right)^{2}}{\mu_{x}}=\omega^{2} \varepsilon_{z} .
\end{aligned}
$$

Based on the dispersion relations of two mediums and the boundary conditions, we can get the reflection coefficient for TE waves

$$
R_{\mathrm{E}}=\frac{\mu_{y} k_{0 x}^{\mathrm{TE}}-\mu_{0} k_{t x}^{\mathrm{TE}}}{\mu_{y} k_{0 x}^{\mathrm{TE}}+\mu_{0} k_{t x}^{\mathrm{TE}}} .
$$

When $k_{t x}^{\mathrm{TE}}$ becomes imaginary, that is,

$$
\frac{\mu_{y}}{\mu_{x}}\left(k_{y}^{\mathrm{TE}}\right)^{2}>\omega^{2} \varepsilon_{z} \mu_{y} \text {, }
$$

total reflection occurs at the boundary. Together with (3), we can get the total reflection conditions in Table 1.

Generally, we choose two biaxial anisotropic mediums: medium 1 is left-handed medium with the constitutive parameters: $\overline{\bar{\varepsilon}}_{1}=\left[\begin{array}{ccc}0.6 & 0 & 0 \\ 0 & 0.5 & 0 \\ 0 & 0 & -0.5\end{array}\right] ; \overline{\bar{\mu}}_{1}=\left[\begin{array}{ccc}-0.5 & 0 & 0 \\ 0 & -0.9 & 0 \\ 0 & 0 & 0.7\end{array}\right]$; medium 2 is right-handed medium with the constitutive parameters: $\overline{\bar{\varepsilon}}_{2}=\left[\begin{array}{ccc}0.6 & 0 & 0 \\ 0 & 0.5 & 0 \\ 0 & 0 & 1\end{array}\right] ; \overline{\bar{\mu}}_{2}=\left[\begin{array}{ccc}0.25 & 0 & 0 \\ 0 & 0.3 & 0 \\ 0 & 0 & 0.2\end{array}\right]$. Both of them satisfy the condition b of case (A) shown in Table 1 and the critical angles are both $\theta_{c}=30^{\circ}$. In Figure 2, we show $z$ component of electric field in the $x y$ plane generated by the TE incident wave with two different incident angles. The incident angles in Figures 2(a) and 2(c) are both $25^{\circ}$. We can see that there is nontotal reflection. Differently, the transmission wave and the incident wave are on the same side of the normal in medium 1 (left-handed) while they are on the different side in the 


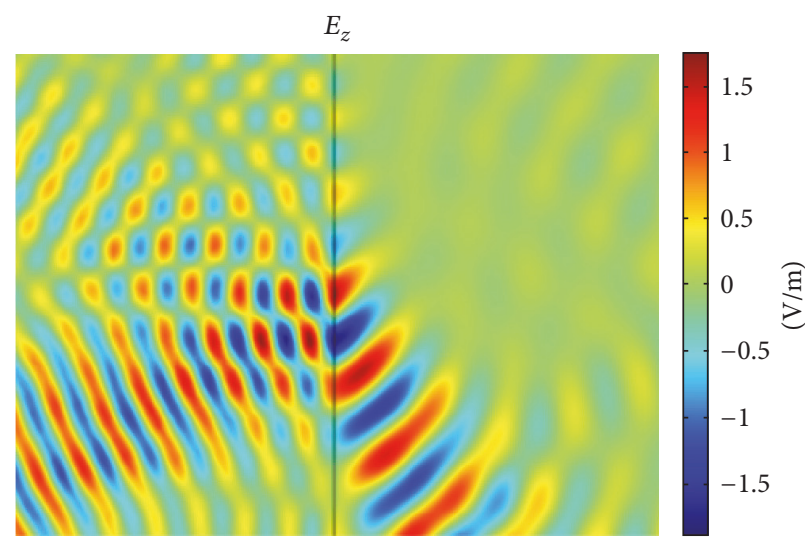

(a)

$E_{z}$

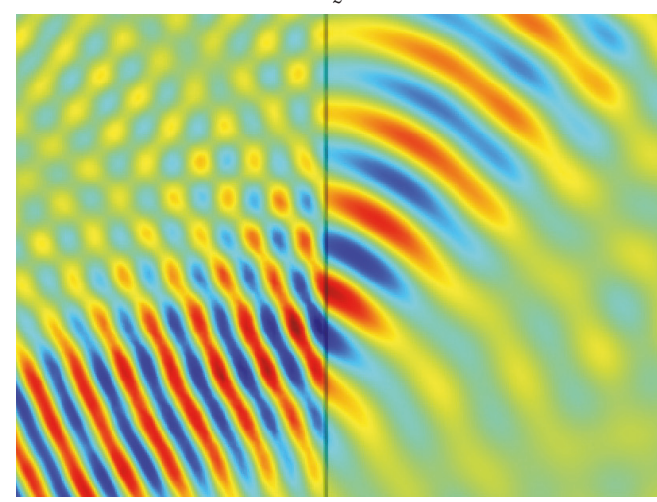

(c)

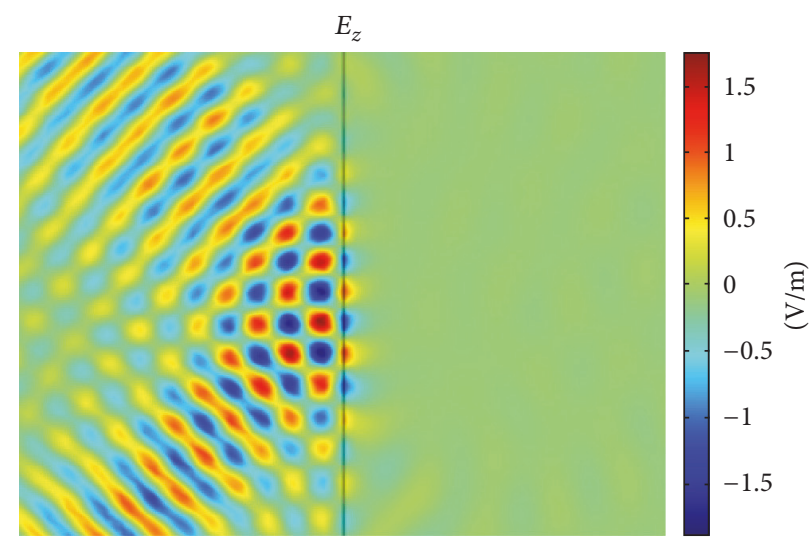

(b)

$E_{z}$

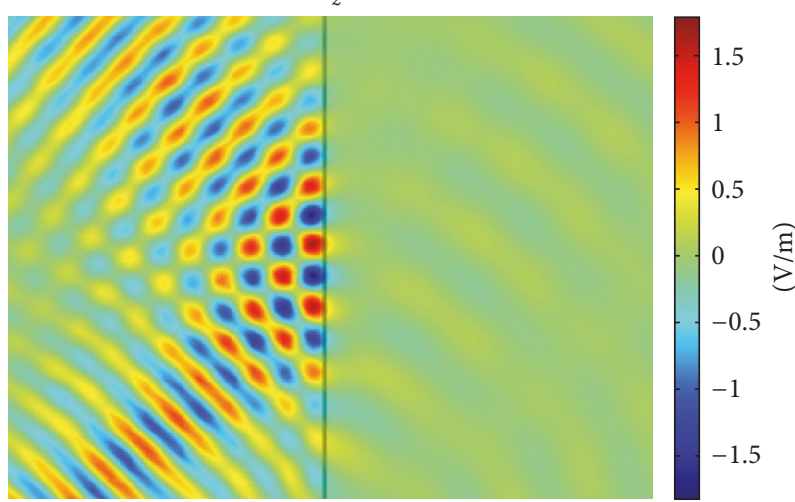

(d)

FIGURE 2: $E_{z}$ of a TE wave incident from isotropic medium into biaxial anisotropic medium; (a) medium 1 (left-handed) with incident angle $\theta_{i}=25^{\circ}$; (b) medium 1 (left-handed) with $\theta_{i}=45^{\circ}$, total reflection; (c) medium 2 (right-handed) with $\theta_{i}=25^{\circ}$; (d) medium 2 (right-handed) with $\theta_{i}=45^{\circ}$, total reflection.

medium 2 (right-handed). Shown as Figures 2(b) and 2(d), when the incident angles are $45^{\circ}\left(\theta_{i}>\theta_{c}\right)$, total reflection occurs at the boundary. All the results are in accordance with the theoretical derivation summarized in Table 1.

2.2. Total Transmission of TM Waves. Similarly, consider a TM incident case. According to the dispersion relations of TM waves in two mediums,

$$
\begin{aligned}
& \left(k_{0 x}^{\mathrm{TM}}\right)^{2}+\left(k_{y}^{\mathrm{TM}}\right)^{2}=\omega^{2} \varepsilon_{0} \mu_{0}, \\
& \frac{\left(k_{x}^{\mathrm{TM}}\right)^{2}}{\varepsilon_{y}}+\frac{\left(k_{y}^{\mathrm{TM}}\right)^{2}}{\varepsilon_{x}}=\omega^{2} \mu_{z},
\end{aligned}
$$

and the boundary conditions, the reflection coefficient for TM waves can be written as

$$
R_{M}=\frac{\varepsilon_{y} k_{0 x}^{\mathrm{TM}}-\varepsilon_{0} k_{t x}^{\mathrm{TM}}}{\varepsilon_{y} k_{0 x}^{\mathrm{TM}}+\varepsilon_{0} k_{t x}^{\mathrm{TM}}} .
$$

When total transmission occurs at the boundary, let $R_{M}=$ 0 ; we can get the total transmission condition in terms of Brewster Angle $\theta_{B}$

$$
\sin ^{2} \theta_{B}=\frac{\varepsilon_{x}\left(\mu_{0} \varepsilon_{y}-\mu_{z} \varepsilon_{0}\right)}{\mu_{0}\left(\varepsilon_{x} \varepsilon_{y}-\varepsilon_{0}^{2}\right)} .
$$

Since $0<\sin ^{2} \theta_{B}<1$, the existence conditions of Brewster Angle $\theta_{B}$ can be written as follows:

$$
\begin{aligned}
\text { If } \varepsilon_{x}>0, & \\
& \left(\mu_{0} \varepsilon_{y}-\mu_{z} \varepsilon_{0}\right) \mu_{0}\left(\varepsilon_{x} \varepsilon_{y}-\varepsilon_{0}^{2}\right)>0 \\
& \left(\mu_{z} \varepsilon_{x}-\varepsilon_{0} \mu_{0}\right) \mu_{0}\left(\varepsilon_{x} \varepsilon_{y}-\varepsilon_{0}^{2}\right)>0 . \\
\text { If } \varepsilon_{x}<0, & \\
& \left(\mu_{0} \varepsilon_{y}-\mu_{z} \varepsilon_{0}\right) \mu_{0}\left(\varepsilon_{x} \varepsilon_{y}-\varepsilon_{0}^{2}\right)<0 \\
& \left(\mu_{z} \varepsilon_{x}-\varepsilon_{0} \mu_{0}\right) \mu_{0}\left(\varepsilon_{x} \varepsilon_{y}-\varepsilon_{0}^{2}\right)<0 .
\end{aligned}
$$

We can see that total transmission at the boundary between an isotropic medium and a biaxial anisotropic medium for 
TABLE 2: Conditions of total transmission of TM waves.

\begin{tabular}{ll}
\hline The range of constitutive parameters & The condition of total transmission \\
\hline (D) $\frac{\varepsilon_{y}}{\mu_{z}}=\frac{\varepsilon_{0}}{\mu_{0}} \cap \varepsilon_{x} \varepsilon_{y} \neq \varepsilon_{0}^{2}$ & Total transmission occurs only when TM waves are incident vertically \\
(E) $\frac{\varepsilon_{y}}{\mu_{z}}=\frac{\varepsilon_{0}}{\mu_{0}} \cap \varepsilon_{x} \varepsilon_{y}=\varepsilon_{0}^{2}$ & Total transmission occurs for all TM waves no matter what incident angle $\theta_{i}$ is \\
(F) $\frac{\varepsilon_{y}}{\mu_{z}} \neq \frac{\varepsilon_{0}}{\mu_{0}} \cap \varepsilon_{x} \varepsilon_{y} \neq \varepsilon_{0}^{2}$ & Total transmission occurs only when the incident angle is Brewster Angle $\theta_{B}$ \\
\hline
\end{tabular}

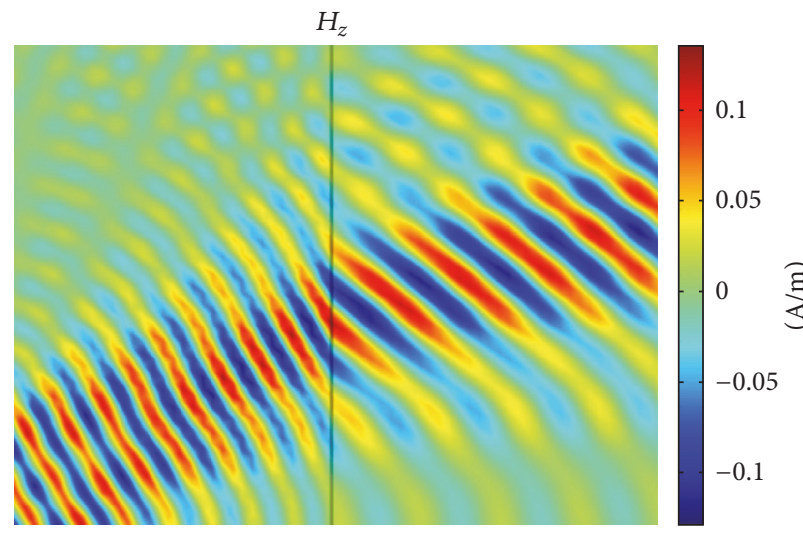

(a)

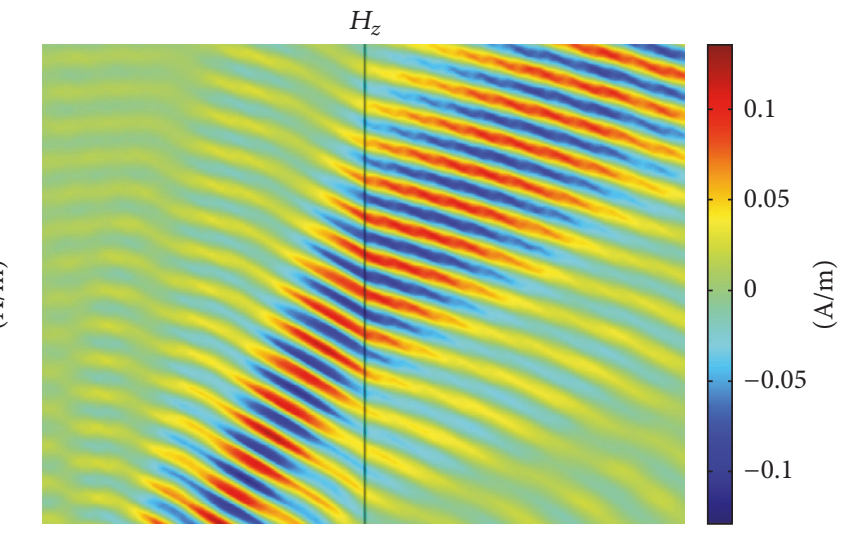

(b)

Figure 3: $H_{z}$ of a TM wave incident from isotropic medium into biaxial anisotropic medium, total transmission occurs. (a) The incident angle $\theta_{i}=30^{\circ}$. (b) $\theta_{i}=60^{\circ}$.

TM waves is up to the value of $\varepsilon_{x}, \varepsilon_{y}$, and $\mu_{z}$. Together with (7), we can get the total transmission conditions in Table 2.

Generally, we choose the constitutive parameters of biaxial anisotropic medium to match condition (E) in Table 2: $\overline{\bar{\varepsilon}}=\left[\begin{array}{ccc}2 & 0 & 0 \\ 0 & 0.5 & 0 \\ 0 & 0 & 0.6\end{array}\right] ; \overline{\bar{\mu}}=\left[\begin{array}{ccc}0.9 & 0 & 0 \\ 0 & 0.8 & 0 \\ 0 & 0 & 0.5\end{array}\right]$. In Figure 3, we show $z$ component of magnetic field with a TM incidence with two different incident angles. The incident angles are $30^{\circ}$ and $60^{\circ}$, respectively. We can see that, for both cases, total transmission occurs at the boundary. All the results match with the theoretical derivation listed in Table 2.

Besides, in order to demonstrate the condition that total transmission occurs only when the incident angle is Brewster Angle $\theta_{B}$, we choose condition (F) in Table 2: $\overline{\bar{\varepsilon}}=\left[\begin{array}{ccc}2 & 0 & 0 \\ 0 & 0.5 & 0 \\ 0 & 0 & 0.6\end{array}\right]$; $\overline{\bar{\mu}}=\left[\begin{array}{ccc}0.9 & 0 & 0 \\ 0 & 0.8 & 0 \\ 0 & 0 & 0.5\end{array}\right]$. Its Brewster Angle $\theta_{B}=50.63^{\circ}$. In Figure 4, we show the $z$ component of magnetic field with a TM incidence with different incident angles. The incident angles are $30^{\circ}, 50.63^{\circ}$, and $70^{\circ}$, respectively. We can see that total transmission only occurs when the incident angle is Brewster Angle $\theta_{B}=50.63^{\circ}$. All the results match with the theoretical derivation listed in Table 2.

Other situations can also be simulated and verified.

\section{Result and Discussion}

Since biaxial anisotropic medium is a reciprocal medium, if total transmission occurs at the boundary when a TM wave is incident from isotropic medium into biaxial anisotropic medium, total transmission will also occur when this TM wave is incident from biaxial anisotropic medium into the same isotropic medium. Therefore, TM waves can full-pass the biaxial anisotropic slab, independent of the thickness of the slab as long as constitutive parameters match the condition in Table 2.

Besides, the dispersion relations of TE waves and TM waves are dual (according to (4) and (8)), so we can get the other dispersion relation by exchanging the elements of $\overline{\bar{\varepsilon}}$ and $\overline{\bar{\mu}}$ in either relation $\left(\mu_{x}, \mu_{y}\right.$, and $\varepsilon_{z}$ exchange with $\varepsilon_{x}, \varepsilon_{y}$, and $\mu_{z}$ ). Also because of the dual property, although we only discussed the total reflection with TE waves incidence above, we can get the TM case if we replace $\mu_{x}, \mu_{y}$, and $\varepsilon_{z}$ with $\varepsilon_{x}, \varepsilon_{y}$, and $\mu_{z}$. In the similar way, for total transmission, we can we can get the condition for TE waves by replacing $\varepsilon_{x}, \varepsilon_{y}$, and $\mu_{z}$ with $\mu_{x}, \mu_{y}$, and $\varepsilon_{z}$.

Hence, if biaxial anisotropic medium's permittivity $\overline{\bar{\varepsilon}}$ and permeability $\overline{\bar{\mu}}$ are set appropriately for some special value, it will simultaneously satisfy both the condition of TE (TM) waves' total reflection and TM (TE) waves' total transmission. In this way, the separation of TE waves and TM waves can be realized easily. Specifically, there are two ways to design such a wave splitter.

3.1. Total Reflection for TE Waves and Total Transmission for TM Waves. We can choose constitutive parameters to satisfy one of the conditions in Tables 1 and 2 to separate TE waves and TM waves, which are combined both in incidence waves. In this way, TE waves will totally reflect while TM waves will totally transmit. 


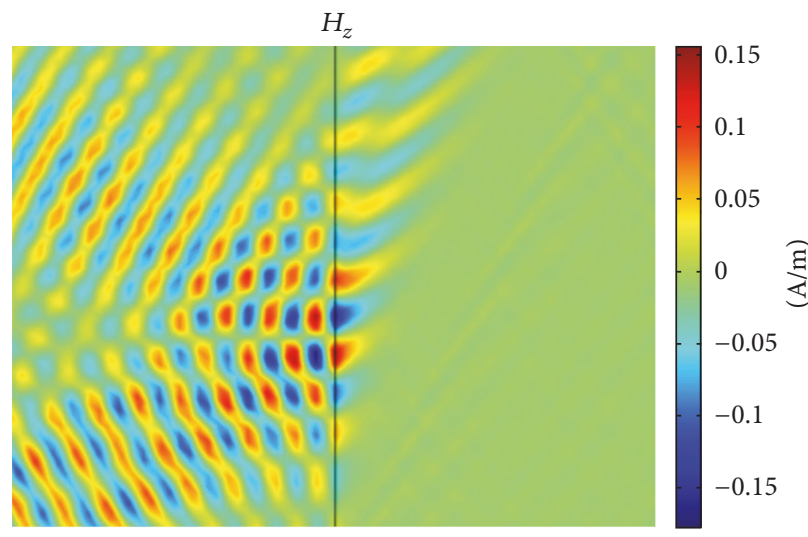

(a) $\theta_{i}=30^{\circ}$

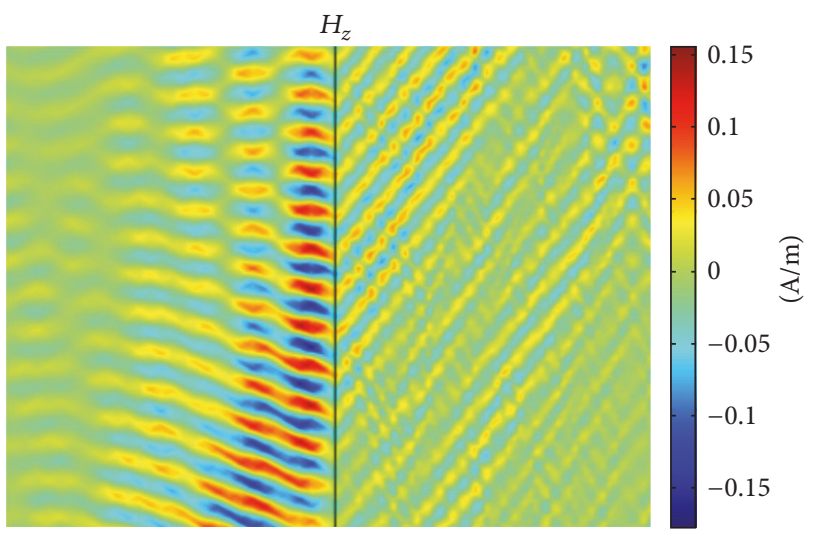

(b) $\theta_{i}=70^{\circ}$

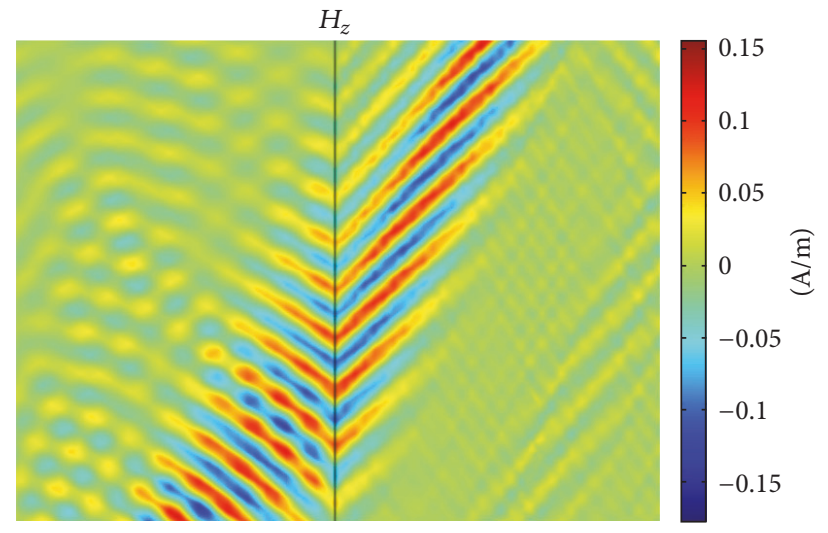

(c) $\theta_{i}=\theta_{B}=50.63^{\circ}$

Figure 4: $H_{z}$ of a TM wave incident from isotropic medium into biaxial anisotropic medium. (a) The incident angle $\theta_{i}=30^{\circ}$, no total transmission. (b) $\theta_{i}=70^{\circ}$, no total transmission. (c) The incident angle is Brewster Angle $\theta_{B}=50.63^{\circ}$, total transmission occurs.

$E_{z}$

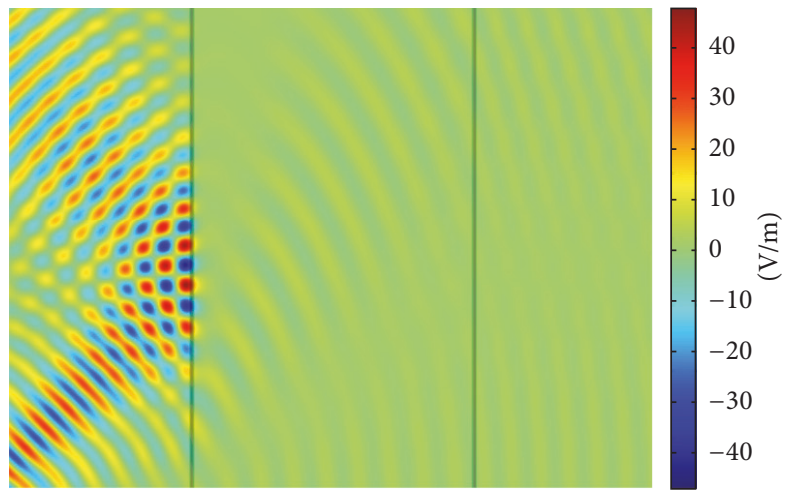

(a)

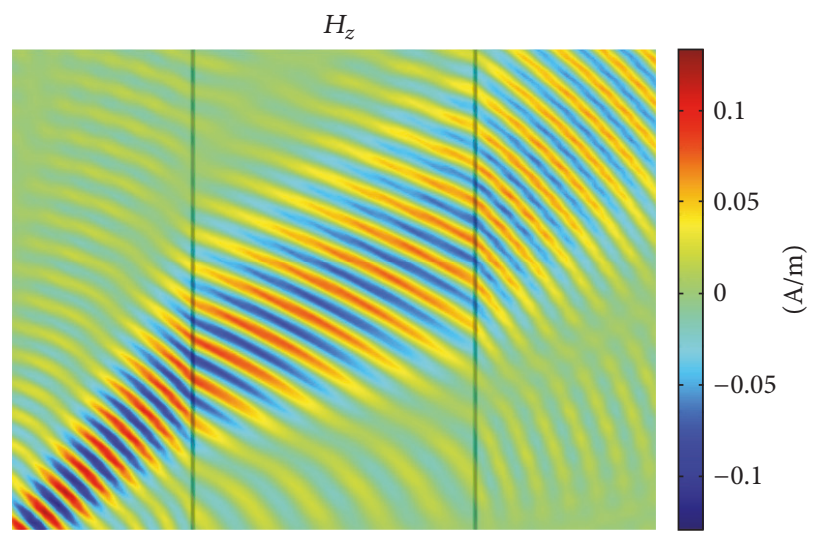

(b)

FIGURE 5: The total reflection for TE waves and total transmission for TM waves of a Gaussian beam incident from air into a biaxial anisotropic slab with the incident angle $\theta_{i}=45^{\circ}$. (a) $E_{z}$ of an incident TE wave, total reflection. (b) $H_{z}$ of an incident TM wave, total transmission.

For instance, we choose biaxial anisotropic medium's constitutive parameters to satisfy condition (b) of case (A) in Table 1 and case (E) in Table 2: $\overline{\bar{\varepsilon}}=\left[\begin{array}{ccc}2 & 0 & 0 \\ 0 & 0.5 & 0 \\ 0 & 0 & 1\end{array}\right] ; \overline{\bar{\mu}}=$ $\left[\begin{array}{ccc}0.25 & 0 & 0 \\ 0 & 0.8 & 0 \\ 0 & 0 & 0.5\end{array}\right]$; in this way, its critical angle is $\theta_{c}=30^{\circ}$ and the total reflection condition of TE waves is satisfied. In Figure 5, we show the electric field and the magnetic field when a Gaussian beam is incident from air into the biaxial slab with the incident angle of $45^{\circ}$. We can see from Figure 5(a) that when $\theta_{i}>\theta_{c}$, total reflection occurs for TE 


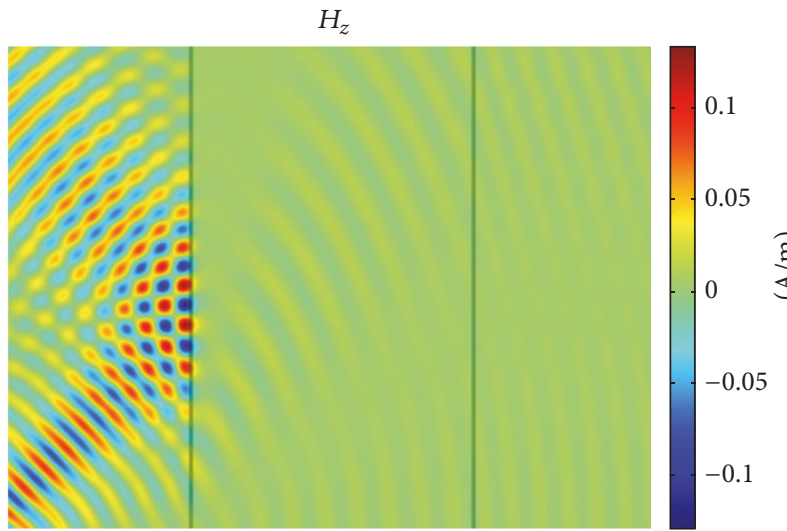

(a)

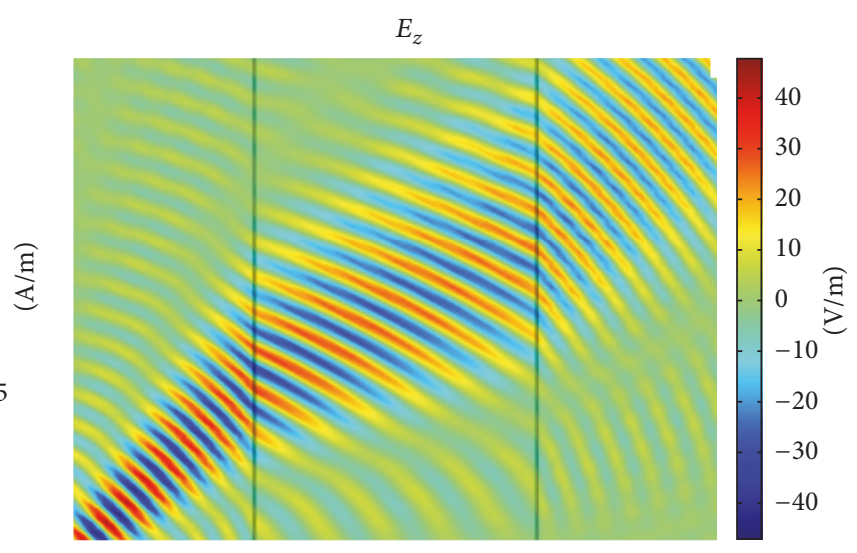

(b)

FIGURE 6: The total reflection for TM waves and total transmission for TE waves of a Gaussian beam incident from air into a biaxial anisotropic slab with the incident angle $\theta_{i}=45^{\circ}$. (a) $H_{z}$ of an incident TM wave, total reflection. (b) $E_{z}$ of an incident TE wave, total transmission.

waves. Figure 5(b) shows that TM waves can totally transmit.

3.2. Total Reflection for TM Waves and Total Transmission for TE Waves. For this case, we choose biaxial anisotropic medium's constitutive parameters: $\overline{\bar{\varepsilon}}=\left[\begin{array}{ccc}-0.5 & 0 & 0 \\ 0 & -0.7 & 0 \\ 0 & 0 & 0.5\end{array}\right] ; \overline{\bar{\mu}}=$ $\left[\begin{array}{ccc}2 & 0 & 0 \\ 0 & 0.5 & 0 \\ 0 & 0 & 0.8\end{array}\right]$. These constitutive parameters satisfy both the condition of TM waves' total reflection and TE waves' total transmission. Figure 6(a) shows that there is total reflection for TM waves. Figure 6(b) shows that there is always total transmission for TE waves.

\section{Conclusion}

This paper firstly discusses the total reflection and total transmission when electromagnetic waves are incident from a semi-infinite isotropic medium into a biaxial anisotropic medium. Furthermore, we design a TE/TM wave splitter based on a biaxial anisotropic slab. The structure of the wave splitter is simple and has little limitation on constitutive parameters, incident angle, and chirality. Meanwhile, such wave splitters can separate TE waves and TM waves as long as constitutive parameters are chosen in a wide range. The results of simulation verify the theoretical derivations.

\section{Conflicts of Interest}

The authors declare that there are no conflicts of interest regarding the publication of this paper.

\section{Acknowledgments}

This work is supported by the Fundamental Research Funds for the Central Universities (2014JBZ017).

\section{References}

[1] V. G. Veselago, "The electrodynamics of substances with simultaneously negative values of $\varepsilon$ and $\mu$," Soviet Physics Uspekh, vol. 10, no. 4, pp. 509-514, 1968.

[2] D. R. Smith, W. J. Padilla, D. C. Vier, S. C. Nemat-Nasser, and S. Schultz, "Composite medium with simultaneously negative permeability and permittivity," Physical Review Letters, vol. 84, no. 18, pp. 4184-4187, 2000.

[3] J. A. Kong, Electromagnetic Wave Theory, Cambridge, New York, NY, USA, 2008.

[4] C.-W. Qiu, H.-Y. Yao, L.-W. Li, S. Zouhdi, and T.-S. Yeo, "Routes to left-handed materials by magnetoelectric couplings," Physical Review B, vol. 75, no. 24, Article ID 245214, 2007.

[5] J. B. Pendry, "A chiral route to negative refraction," Science, vol. 306, no. 5700, pp. 1353-1355, 2004.

[6] L. Hu and S. T. Chui, "Characteristics of electromagnetic wave propagation in uniaxially anisotropic left-handed materials," Physical Review B, vol. 66, no. 8, Article ID 085108, 2002.

[7] X. Cheng, H. Chen, B.-L. Wu, and J. A. Kong, "Visualization of negative refraction in chiral nihility media," IEEE Antennas and Propagation Magazine, vol. 51, no. 4, pp. 79-87, 2009.

[8] S. Tretyakov, I. Nefedov, A. Sihvola, S. Maslovski, and C. Simovski, "Waves and energy in chiral nihility," Journal of Electromagnetic Waves and Applications, vol. 17, no. 5, pp. 695706, 2003.

[9] J.-F. Dong and J. Li, "The reflection and transmission of electromagnetic waves by a uniaxial chiral slab," Progress in Electromagnetics Research, vol. 127, pp. 389-404, 2012.

[10] L. XianGang, "Principles of electromagnetic waves in metasurfaces," Science China Physics, Mechanics \& Astronomy, vol. 58, Article ID 594201, 2015.

[11] X. Luo, M. Pu, X. Ma, and X. Li, “Taming the electromagnetic boundaries via metasurfaces: from theory and fabrication to functional devices," International Journal of Antennas and Propagation, vol. 2015, Article ID 204127, 2015.

[12] X. Ling, X. Zhou, X. Yi et al., "Giant photonic spin Hall effect in momentum space in a structured metamaterial with spatially varying birefringence," Light: Science \& Applications, vol. 4, no. 5, article e290, 2015. 
[13] J. Wang, H. Huang, C. Chen, H. He, Y. Dong, and H. Qi, "Goos-Hänchen lateral displacements at the interface between isotropic and gyroelectric media," International Journal of Antennas and Propagation, vol. 2013, Article ID 596278, 2013.

[14] A. Polemi, S. Maci, and P.-S. Kildal, "Dispersion characteristics of a metamaterial-based parallel-plate ridge gap waveguide realized by bed of nails," IEEE Transactions on Antennas and Propagation, vol. 59, no. 3, pp. 904-913, 2011.

[15] S. A. Ramakrishna, "Physics of negative refractive index materials," Reports on Progress in Physics, vol. 68, no. 2, pp. 449-521, 2005.

[16] N. I. Landy, S. Sajuyigbe, J. J. Mock et al., "Perfect metamaterial absorber," Physical Review Letters, vol. 100, no. 20, Article ID 207402, pp. 1-4, 2008.

[17] H. Luo, Z. Ren, W. Shu, and F. Li, "Construct a polarizing beam splitter by an anisotropic metamaterial slab," Applied Physics B: Lasers and Optics, vol. 87, no. 2, pp. 283-287, 2007.

[18] T. Jiang, Y. Chen, and Y.-J. Feng, "Subwavelength rectangular cavity partially filled with left-handed materials," Chinese Physics, vol. 15, no. 6, article 006, pp. 1154-1160, 2006.

[19] I. V. Lindell, S. A. Tretyakov, K. I. Nikoskinen, and S. Ilvonen, "BW media-media with negative parameters, capable of supporting backward waves," Microwave and Optical Technology Letters, vol. 31, no. 2, pp. 129-133, 2001.

[20] H. Luo, Z. Ren, W. Shu, and F. Li, "Wave propagation in an anisotropic metamaterial with single-sheeted hyperboloid dispersion relation," Applied Physics A: Materials Science and Processing, vol. 87, no. 2, pp. 245-249, 2007.

[21] Y.-Y. Jiang, H.-Y. Shi, Y.-Q. Zhang, C.-F. Hou, and X.-D. Sun, "Characteristics of surface waves in anisotropic left-handed materials," Chinese Physics, vol. 16, no. 7, article 026, pp. 19591962, 2007.

[22] X. Cheng, H. Chen, L. Ran, B.-I. Wu, T. M. Grzegorczyk, and J. A. Kong, "Negative refraction and cross polarization effects in metamaterial realized with bianisotropic S-ring resonator," Physical Review B - Condensed Matter and Materials Physics, vol. 76, no. 2, Article ID 024402, 2007.

[23] M. R. Jalal and F. V. Alamdarlo, "Simulation of light interference by a biaxial thin film," Optik-International Journal for Light and Electron Optics, vol. 130, pp. 393-397, 2017.

[24] M. Cheng and R. Chen, "Large positive and negative lateral shifts from an anisotropic metamaterial slab backed by a metal," Chinese Physics Letters, vol. 26, no. 1, Article ID 014101, 2009.

[25] Y. H. Guo, L. S. Yan, W. Pan et al., "A plasmonic splitter based on slot cavity," Optics Express, vol. 19, no. 15, pp. 13831-13838, 2011.

[26] Y. Guo, L. Yan, W. Pan et al., "Characteristics of plasmonic filters with a notch located along rectangular resonators," Plasmonics, vol. 8, no. 2, pp. 167-171, 2013.

[27] H. Huang, Y. Fan, B.-I. Wu, and J. A. Kong, “Tunable TE/TM wave splitter using a gyrotrppic slab," Progress in Electromagnetics Research-Pier, vol. 85, pp. 367-380, 2008.

[28] J. Zhou, W. Zhang, Y. Liu et al., "Spin-dependent manipulating of vector beams by tailoring polarization," Scientific Reports, vol. 6, no. 1, Article ID 34276, 2016. 


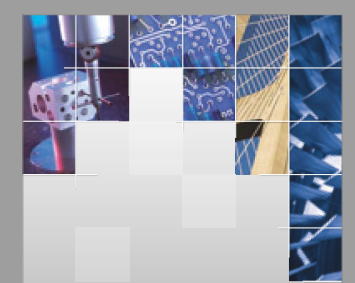

\section{Enfincering}
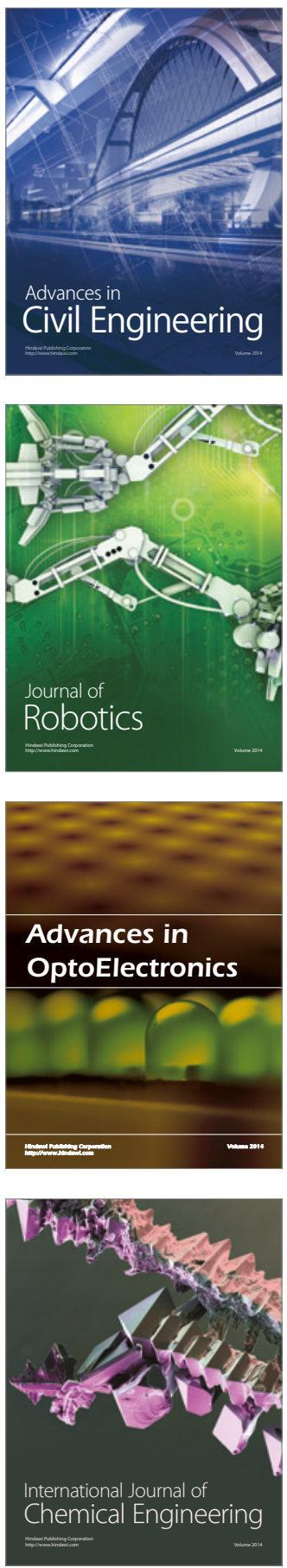

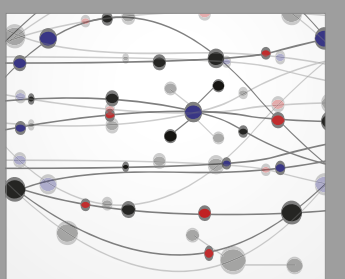

The Scientific World Journal

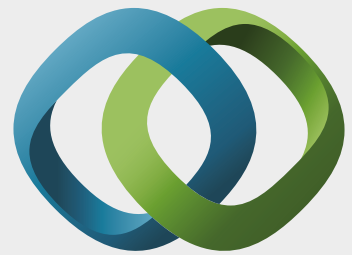

\section{Hindawi}

Submit your manuscripts at

https://www.hindawi.com
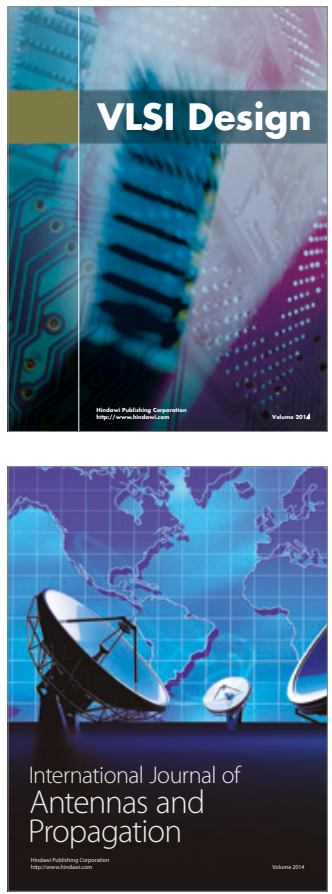

\section{Rotating}

Machinery
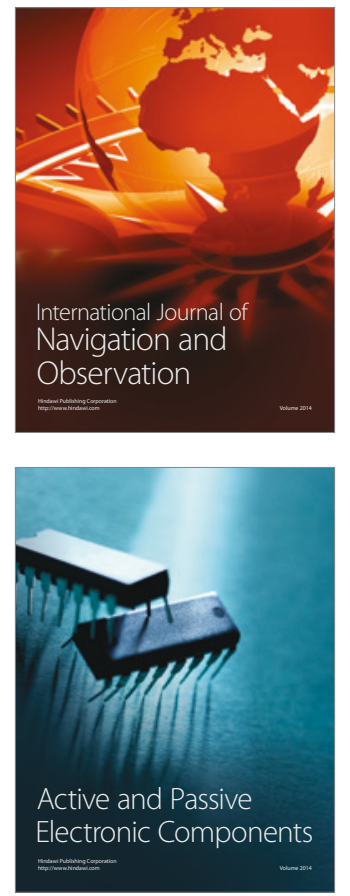
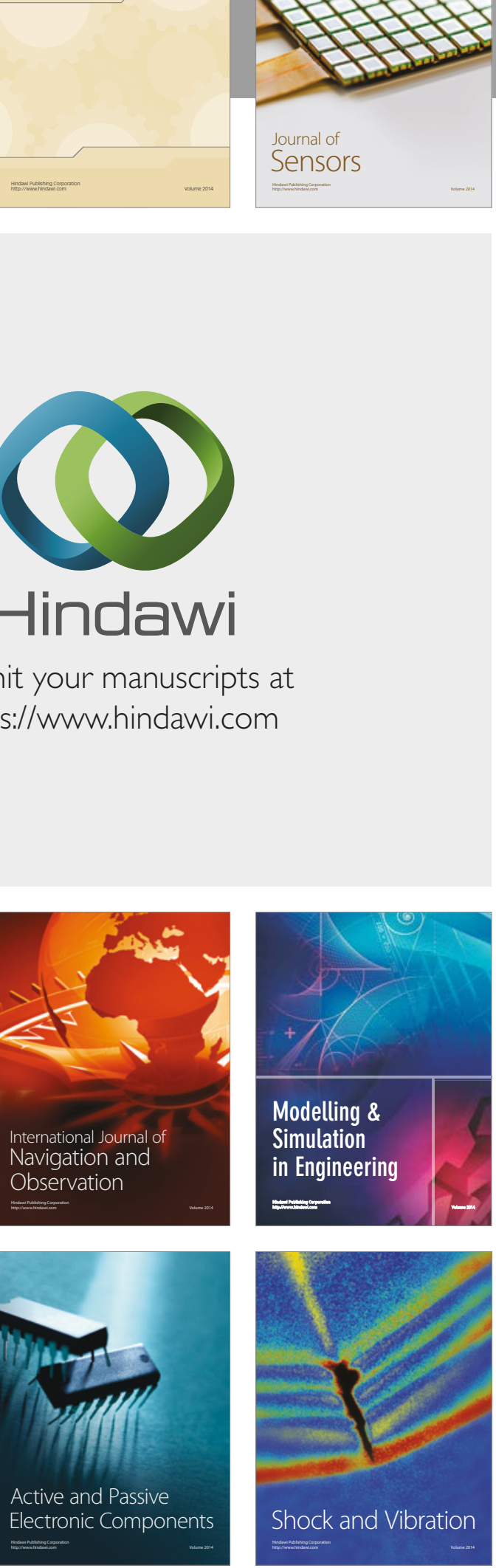
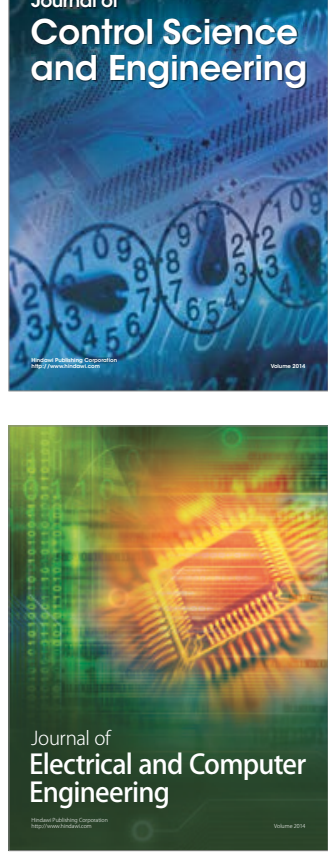

Distributed

Journal of

Control Science

and Engineering
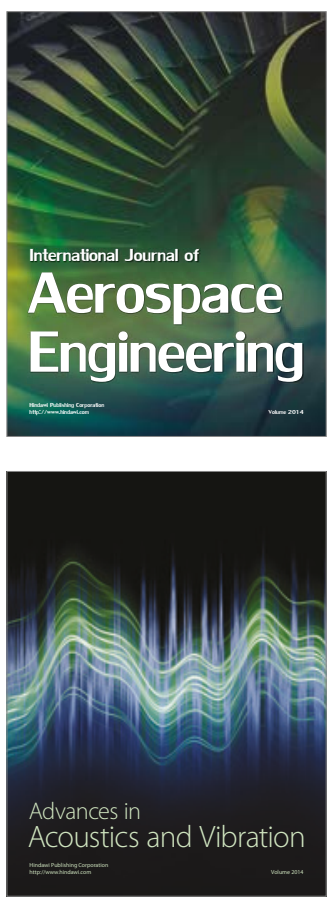

Sensor Networks 